\title{
Candidate biomarkers for cervical cancer treatment: Potential for clinical practice (Review)
}

\author{
MIHO IIDA, KOUJI BANNO, MEGUMI YANOKURA, KANAKO NAKAMURA, MASATAKA ADACHI, \\ YUYA NOGAMI, KIYOKO UMENE, KENTA MASUDA, IORI KISU, \\ TAKASHI IWATA, KYOKO TANAKA and DAISUKE AOKI
}

Department of Obstetrics and Gynecology, School of Medicine, Keio University, Tokyo 160-8582, Japan

Received February 7, 2014; Accepted March 5, 2014

DOI: $10.3892 / \mathrm{mco} .2014 .324$

\begin{abstract}
Cervical cancer ranks high among the causes of female cancer mortalities and is an important disease in developing and developed countries. Current diagnosis of cervical cancer depends on colposcopy, pathological diagnosis and preoperative diagnosis using methods, including magnetic resonance imaging and computed tomography. Advanced cervical cancer has a poor prognosis. The tumor marker squamous cell carcinoma is conventionally used for screening, but recent studies have revealed the mechanisms of carcinogenesis and the factors associated with a poor prognosis in cervical cancer. These include epigenetic biomarkers, with the methylation level of the checkpoint with forkhead and ring finger gene being potentially useful for predicting the malignancy of cervical cancer and sensitivity to treatment with paclitaxel. The extent of methylation of the Werner DNA helicase gene is also useful for determining sensitivity to an anticancer agent, CPT-11. In addition to epigenetic changes, the expression levels of hypoxia-inducible factor $1 \alpha$ subunit, epidermal growth factor receptor and cyclooxygenase- 2 have been reported as possible biomarkers in cervical cancer. Novel prognostic factors, including angiogenic factors, fragile histidine triad, thymidylate synthase, glucose-related protein 58 and mucin antigens, have also been described, and hemoglobin and platelets may also be significant prognostic biomarkers. Utilization of these biomarkers may facilitate personalized treatment and improved outcomes in cervical cancer.
\end{abstract}

Correspondence to: Dr Kouji Banno, Department of Obstetrics and Gynecology, School of Medicine, Keio University, 35 Shinanomachi, Shinjuku-ku, Tokyo 160-8582, Japan

E-mail: kbanno@z7.keio.jp

Key words: cervical cancer, biomarker, checkpoint with forkhead and ring finger, Werner DNA helicase, hypoxia inducible factor $1 \alpha$, epidermal growth factor receptor

\section{Contents}

1. Introduction

2. Biomarkers associated with sensitivity to anticancer agents

3. Biomarkers for the prediction of radiosensitivity

4. Biomarkers in peripheral blood

5. Biomarkers for the prognostic prediction of cervical cancer

6. Conclusion

\section{Introduction}

Cervical cancer is the second leading cause of female cancer mortalities worldwide and 500,000 new cases are diagnosed annually in developing and developed countries. In the United States, there are 12,800 new cases of cervical cancer each year and 4,600 females succumbed to the disease in 2000 (1). In Japan, early-stage cervical cancer is on the increase among females of reproductive age (20-40 years old) and diagnosis and treatment of the disease in this age group is important due to the declining birth rate and aging population (2). The background to these changes may include a decrease in the age of initial sexual activity and exposure to high-risk human papilloma viruses (HPVs).

Unlike the majority of other gynecological malignancies, cervical cancer is clinically staged prior to surgery. Early stages [International Federation of Gynecology and Obstetrics (FIGO) stage IA1-IB2] are often treated surgically, but cervical cancer with distant metastases or recurrence remains uniformly fatal (3). Prognostic factors include the clinical stage and histological cancer type (4). Due to the requirement for implementing personalized treatment and evaluating outcomes, biomarkers for predicting prognosis have emerged from recent studies (5). In addition to squamous cell carcinoma (SCC), a tumor marker conventionally used for cervical cancer, several biomarkers have been identified that predict the response to anticancer therapy, including checkpoint with forkhead and ring finger (CHFR); Werner DNA helicases (WRN); hypoxia-inducible factor-1 $\alpha$ subunit (HIF-1 $\alpha$ ), which is associated with hypoxic response; epidermal growth factor receptor (EGFR), which may be a molecular target; and cyclooxygenase-2 (COX-2), which predicts radiation sensitivity (6). 


\section{Biomarkers associated with sensitivity to anticancer agents}

CHFR is involved in checkpoint regulation of somatic cell division. CHFR serves as a G2/M checkpoint protein for somatic cell division, delays entry into metaphase from antephase (7) and suppresses the activity of Aurora A kinase, which promotes cell cycling downstream of CHFR. Low temperature and microtubular stress have been indicated to be factors activating CHFR. Thus, introduction of the CHFR gene into HeLa cells, a human cervical cancer-derived cell line in which CHFR is inactivated, produced recovery of the normal cell cycle suppression mechanism when cells were exposed to agents with microtubular toxicity. Hypermethylation of $\mathrm{CpG}$ islands in the promoter region leads to the silencing of CHFR. This mechanism involves the activation of DNA methyltransferases, including DNMT1, and DNMTs are commonly overexpressed in human cancer cells. Thus, activation of DNMTs causes CpG islands of CHFR to be hypermethylated, with resultant suppression of CHFR, promotion of cell cycling and subsequent carcinogenesis.

CHFR expression varies depending on the histological type of cervical cancer. In our previous study, aberrant hypermethylation of the CHFR gene was found in 2/12 cervical adenocarcinoma smears (8), compared with no aberrant DNA hypermethylation in normal cervical cells and cervical SCC cells. In SKG-IIIa cells derived from SCC and without aberrant hypermethylation, treatment with paclitaxel alone caused a marked increase in cells in the $\mathrm{G} 2 / \mathrm{M}$ phase to $73.9 \%$,indicating an active repair mechanism in response to damage caused by paclitaxel. By contrast, in HeLa cells with aberrant CHFR hypermethylation, the percentage of $\mathrm{G} 2 / \mathrm{M}$ cells remained at $8.3 \%$ and sub-G cells increased to $13.4 \%$ following paclitaxel treatment, indicating that paclitaxel induced apoptosis of HeLa cells. However, the combined treatment of paclitaxel and 5-aza-deoxycytidine (dC), a demethylation agent, resulted in $73.9 \%$ of HeLa cells in the G2/M phase and a marked decrease in sub-G1 cells to $2.2 \%$. Therefore, if CHFR is active, the cell cycle is delayed and this allows repair of damaged DNA, causing reduced sensitivity to paclitaxel. However, if CHFR is inactive, damaged DNA cannot be repaired and continuation of somatic cell division leads to apoptosis and increased sensitivity to paclitaxel. Cervical adenocarcinoma has lower sensitivity to anticancer agents compared with cervical SCC (9), and has higher sensitivity to these agents if CHFR is epigenetically suppressed (10). Thus, the aberrant hypermethylation of CHFR in cervical adenocarcinoma is a candidate biomarker for sensitivity to paclitaxel.

Following the silencing of DNMT1 in HeLa cells, Zhang et al (11) identified demethylation of cyclin A1 (CCNAI), CHFR, paired box $1(P A X 1)$, secreted frizzled-related protein 4 (SFRP4) and tumor suppressor in lung cancer 1 (TSLC1), and maintenance of the methylation of phosphatase and tensin homolog deleted on chromosome 10 (PTEN) and fragile histidine triad (FHIT) (Table I). By contrast, similar silencing of DNMT1 in SiHa cells, another human cervical cancer-derived cell line, resulted in the demethylation of CCNA1, PTEN, PAX1, SFRP4 and TSLC1, but maintenance of the methylation of FHIT and CHFR (11). These results showed that silencing of DNMT1 does not influence the methylation of CHFR in SiHa
Table I. DNMT1 silencing and the demethylation of genes in HeLa and SiHa cells.

\begin{tabular}{llc}
\hline Cell line & \multicolumn{1}{c}{ Demethylated } & Not demethylated \\
\hline HeLa & CCNA1, CHFR, & PTEN, FHIT \\
& PAX1, SFRP4, TSLC1 & \\
SiHa & CCNA1, PAX1, & CHFR, FHIT \\
& SFRP4, TSLC1, PTEN & \\
\hline
\end{tabular}

CCNA1 cyclin A1; CHFR, checkpoint with forkhead and ring finger; PAX1, paired box 1; SFRP4, secreted frizzled-related protein 4; TSLC1, tumor suppressor in lung cancer 1; PTEN, phosphatase and tensin homolog deleted on chromosome 10; FHIT, fragile histidine triad.

cells, but reduces its methylation in HeLa cells, indicating that the inhibition of DNMT1 may be a target for the treatment of cervical cancer with HPV-18 infection.

Among the DNMT isoforms, functional cooperativity of DNMT1 and DNMT3B is known to promote carcinogenesis (12). Liu et al (13) found that histone deacetylase (HDAC) inhibitors induce apoptosis in cervical cancer cells by suppressing DNMT3B activity. Treatment of HeLa and CaSki cells with a classical HDAC inhibitor, trichostatin A (TSA), at $1 \mu \mathrm{mol} / 1$ resulted in 86 and $76 \%$ of HeLa and CaSki cells, respectively, undergoing apoptosis, whereas $>90 \%$ of normal cells survived. TSA treatment had no significant effect on normal cells, but decreased DNMT3B activity in HeLa and CaSki cells compared with normal cells.

The expression levels of DNMT1 and CHFR may therefore be biomarkers for predicting the malignancy of cervical adenocarcinoma. In cases of SCC without aberrant hypermethylation of the CHFR gene, the expression level of DNMT1 may be an index of malignancy. Since HDAC inhibitors suppress DNMT3B in cancer cells, these agents may be effective for the treatment of cervical cancer.

The $W R N$ gene codes for DNA helicases that are important for maintaining genomic stability, and is also the gene responsible for Werner syndrome, a rare autosomal recessive genetic disorder. Affected individuals are healthy at birth, but aging phenomena, including short stature, skin atrophy, diminished subcutaneous fat and a bald head, develop rapidly from the late adolescence, and age-related diseases, such as type 2 diabetes, osteoporosis and bilateral cataract, also appear with cancer being the leading cause of mortality (14).

In a previous study, we examined the WRN gene in human cervical SCC-derived cell lines, SKG-I, II, IIIA and IIIB, and human cervical adenocarcinoma-derived cell lines, HeLa and TCO-1 (15). Aberrant hypermethylation of the WRN gene was detected in SKG-II and TCO-1 cells, and mRNA and protein levels for WRN were reduced in these two cell lines compared with other cell lines. Following the administration of 5-aza-dC, the expression of WRN mRNA was recovered in SKG-II and TCO-1 cells. The sensitivity of SKG-II and TCO-1 cells to CPT-11 was increased by treatment with small interfering RNA for WRN, while sensitivity to other anticancer agents was not decreased. Suppression of the WRN gene has also been shown to increase the anticancer effects of CPT-11 in HeLa cells derived from cervical and rectal cancer $(16,17)$. 
In 21 primary cervical cancer smears, Masuda et al (15) also found the aberrant hypermethylation of WRN in $45 \%(5 / 11)$ of cases of adenocarcinoma and 20\% (2/10) of SCC. Thus, the aberrant hypermethylation of WRN is a potential biomarker for carcinogenesis and sensitivity to CPT-11 in cervical cancer.

HIF- $1 \alpha$ forms a heterodimer with a $\beta$-subunit and has an essential role in the mammalian hypoxic response. At normal $\mathrm{O}_{2}$ partial pressure, prolyl residues of HIF-1 $\alpha$ are hydroxylated by prolyl hydroxylase (PHD), which is activated by oxygen, with subsequent ubiquitination and degradation of HIF-1 $\alpha$ (18). At low $\mathrm{O}_{2}$ partial pressure, PHD is deactivated and hydroxylation of prolyl residues of HIF-1 $\alpha$ is inhibited, and thus, HIF-1 $\alpha$ is not degraded. HIF-1 $\alpha$ instead migrates to the nucleus, binds to promoter regions referred to as HIF-1 $\alpha$ responsive elements, and promotes the transcription of molecules required for a hypoxic response, including erythropoietin. Hypoxia and malnutrition in tumors are caused by rapid malignant proliferation. However, tumor cells regulate the expression of molecules associated with the promotion of angiogenesis and sugar uptake, cell survival and suppression of apoptosis through the hypoxic response. Thus, the expression of HIF-1 $\alpha$ allows tumor cell proliferation in a hypoxic or nutrient-poor environment, in which even survival is difficult, and the HIF-1 $\alpha$ level is associated with the prognosis (19).

Hypoxia is considered to increase malignancy and resistance to radiochemotherapy (20-22). Even in early-stage cervical cancer, there are a number of newly-formed vessels and stable HIF-1 $\alpha$ expression. The normal uterine cervix is chronically exposed to hypoxia, while HIF-1 $\alpha$ is not degraded and is stably expressed. Such phenomena occur only at an advanced stage in other solid tumors, which explains the high malignancy rate in cervical cancer (23-25). Higher immunostaining of HIF-1 $\alpha$ has also been associated with lower survival rates in cervical adenocarcinoma and cervical SCC (26-28). In HeLa and SiHa cells, Bai et al (29) identified the overexpression of survivin, in addition to HIF-1 $\alpha$, under hypoxia. Survivin is an apoptosis-suppressing protein that is associated with the regulation of cell proliferation. The expression of survivin is a poor prognostic factor since it is involved in the proliferation of cancer cells and drug resistance (26-30). Following the induction of HIF-1 $\alpha$ by hypoxia, Bai et al (29) demonstrated an upregulated survivin expression that suppressed apoptosis of cervical cancer cells, suggesting that survivin and HIF-1 $\alpha$ are novel therapeutic targets. Among other factors downstream of HIF-1 $\alpha$, Luczak et al (31) found that chemokine receptor 4 (CXCR4) levels in 30 cervical cancer samples were significantly higher compared with normal samples.

Drugs targeting HIF-1 $\alpha$ include topotecan (TPT), which inhibits HIF-1 $\alpha$ (32-35) by targeting topoisomerase I (Top1) and forming a Top1-DNA complex that exerts a cytotoxic effect by breaking double-stranded DNA during replication. TPT-dependent inhibition of HIF- $1 \alpha$ accumulation does not occur if RNA transcription is inhibited, indicating that the effect of TPT requires Topl (33). Phase I and II trials of TPT in cervical cancer are ongoing. Thus, HIF- $1 \alpha$ is a major candidate prognostic biomarker and a target for treatment.

The EGFR gene maps to chromosome 7p11.2-p12 and has 28 exons. The gene encodes a protein that contains an extracellular ligand-binding domain, a transmembrane domain and a tyrosine kinase domain (36). The EGFR family is composed of four heterodimer receptors, EGFR/v-erb-a erythroblastic leukemia viral oncogene homolog 4-1 (ErbB-1), HER2/ErbB-2, HER3/ErbB-3 and HER4/ErbB-4. Heterodimers of EGFR and HER2 (Erb-2) are associated with human carcinogenesis. The signaling pathway of EGFR may be dysregulated in cancer and is associated with carcinogenesis and tumor growth (37-39). In cervical cancer, EGFR is a potential prognostic biomarker, since the coexpression of C-erbB-2 and EGFR is associated with a poor response to chemoradiation (6). In a study of the ectodomain of EGFR in 178 patients with lymph node-negative squamous cell cervical carcinoma following chemoradiotherapy, Halle et al (40) evaluated patient outcomes and the expression of EGFR isoforms using immunohistochemistry. The EGFR isoforms lacked the tyrosine kinase domain, but had the ectodomain. Their expression correlated with the activation of v-Myc myelocytomatosis viral oncogene homolog avian (MYC) and MYC-associated factor X, and with the activation of carcinogenic signaling. The amplification of the EGFR gene is also associated with adverse clinical outcomes in cervical SCC. Thus, Iida et al (41) found significant amplification of the EGFR locus in six of 59 cases of cervical SCC, but in none of 52 cases of adeno/adenosquamous cell carcinoma, with EGFR amplification significantly correlated with shorter overall survival rates.

CXCR7 is coexpressed with EGFR and is a candidate prognostic factor (42). Schrevel et al (42) showed that the expression of CXCR7 occurred more frequently in SCC compared with adeno/adenosquamous cell carcinoma and was significantly associated with tumor size, lymph node metastasis and EGFR expression. Thus, it was concluded that CXCR7 and EGFR are potential therapeutic targets. In a phase II study comparing pazopanib and lapatinib targeting EGFR and HER2 in 152 patients with cervical cancer, patients receiving pazopanib $(n=74)$ had a significantly longer progression-free survival [hazard ratio, 0.66; 90\% confidence interval (CI), 0.48-0.91; $\mathrm{P}=0.013$ ] and overall survival (hazard ratio, 0.67; 90\% CI, 0.46-0.99; $\mathrm{P}=0.045)$ compared with the lapatinib group $(n=78)$. Response rates to pazopanib and lapatinib were 9 and $5 \%$, respectively. The only grade 3 adverse event with a rate of $>10 \%$ was diarrhea (11\% with pazopanib and $13 \%$ with lapatinib), and the rates of grade 4 adverse events were 9 and $12 \%$ for pazopanib and lapatinib, respectively (Table II). Thus, Monk et al (43) suggested that the heterodimer receptors, EGFR and HER2, are major therapeutic targets in cervical cancer.

A high expression of the cluster of differentiation 44 isoform 6 (CD44v6) (44), X-ray repair cross-complementing protein 1 (XRCC1) gene polymorphism (45) and a high level of phosphorylated mTOR (46) is associated with a poor response of cervical cancer to chemotherapy with platinum-based agents. However, the majority of these studies have focused on neoadjuvant chemotherapy.

\section{Biomarkers for the prediction of radiosensitivity}

Of the two isoforms (COX-1 and COX-2) of cyclooxygenase, COX-2 is induced by cytokines and mitogens at inflamed sites and in tumor tissue (47-49). The substrate of COX-2, arachidonic acid, is converted to prostaglandin G2 (PGG2) or PGH2, which are then converted to PGE2, PGD2, PGF2 $\alpha$, PGI2 and thromboxane A2 (TxA2) by PG synthases. PGE2 is associated 
Table II. Effects of pazopanib and lapatinib on cervical cancer.

\begin{tabular}{lcccc}
\hline Treatments & $\begin{array}{c}\text { OS median } \\
\text { (week) }\end{array}$ & $\begin{array}{c}\text { PFS median } \\
\text { (week) }\end{array}$ & $\begin{array}{c}\text { Incidence of G3 } \\
\text { adverse events (\%) }\end{array}$ & $\begin{array}{c}\text { Incidence of G4 } \\
\text { adverse events (\%) }\end{array}$ \\
\hline Pazopanib & 50.7 & 18.1 & 42 & 12 \\
Lapatinib & 39.1 & 17.1 & 32 & 9 \\
\hline
\end{tabular}

OS, overall survival; PFS, progression-free survival.

with carcinogenesis through signaling via G-protein-coupled eicosanoid receptors 1-4, which also involves $\mathrm{Ca}^{2+}$, cyclic adenosine monophosphate, protein kinase $\mathrm{A}$ and phosphoinositide 3-kinase (PI3K). Overexpression of COX-2 in cervical cancer is associated with the inhibition of apoptosis and promotion of angiogenesis (50). Activation of the $\mathrm{PI} 3 \mathrm{~K} / \mathrm{Akt} / \mathrm{COX}-2$ pathway induces resistance to radiation in HeLa cells and the inhibition of COX-2 increases the radiosensitivity of cervical cancer $(51,52)$, as shown using a COX-2 inhibitor, celecoxib (53). Associations between the intensity of immunostaining and reduced survival rates have been reported (54-56) and Kim et al (55) found overall 5-year actuarial survival rates of $57 \%$ for COX-2-positive patients and $83 \%$ for COX-2-negative patients, regardless of the histological type. Thus, COX-2 is a candidate biomarker for prognostic prediction and the prediction of radiosensitivity in cervical cancer (Table III).

Thymidylate synthase (TS) catalyzes the methylation of deoxyuridine monophosphate in de novo pyrimidine synthesis. Additionally, TS expression is an index of the proliferation potential of cells and the biological malignancy of cancer. Suzuki et al (57) produced a highly specific anti-TS polyclonal antibody for an immunohistochemical assay of TS levels in cervical cancer tissues and found that TS was localized in the epithelial cytoplasm and that the stroma was negative. The 5-year survival rate of $87.2 \%$ in 36 patients with low TS expression was significantly improved compared with $36.8 \%$ in 30 patients with a high TS expression. Thus, a high TS expression may affect cell proliferation and migration, invasion and tumor proliferation, and may be a prognostic factor in advanced cervical cancer. High TS levels also reduce radiosensitivity and serve as a useful index for radiation treatment planning.

Kawanaka et al (58) identified the expression of HIF-2 $\alpha$ in tumor-infiltrating macrophages in $72.6 \%$ of patients with primary advanced cervical SCC, and found that a high HIF- $2 \alpha$-positive cell count increased the risk of local recurrence following radiotherapy and was associated with a poorer disease-free survival. HIF-2 $\alpha$ and HIF-1 $\alpha$ are associated with angiogenesis in tumors and are closely correlated with the invasion and metastasis of cancer cells $(59,60)$.

\section{Biomarkers in peripheral blood}

The hemoglobin $(\mathrm{Hb})$ level in peripheral blood is a useful clinical measurement that reflects the oxygen status in cancer tissue (61). In a retrospective study comprising 386 patients with advanced cervical cancer, Girinski et al (62) concluded that a threshold $\mathrm{Hb}$ level of $10 \mathrm{~g} / \mathrm{dl}$ is a significant prognostic factor and that improving anemia through blood transfusion during radiation therapy contributes to an improved prognosis. Similarly, in a review of 630 patients with cervical cancer treated with radiotherapy, Thomas (63) found that the average weekly $\mathrm{Hb}$ nadir level, rather than the baseline $\mathrm{Hb}$ level, was a significant prognostic factor, with a cut-off level of $12 \mathrm{~g} / \mathrm{dl}$. Thus, $\mathrm{Hb}$ is a useful prognostic factor in advanced cervical cancer and increasing the $\mathrm{Hb}$ level prior to or during treatment can also improve the long-term prognosis. In patients with cancer without lymph node metastasis, Hernandez et al (64) showed that those patients with peripheral blood platelet (Plt) counts of $\geq 400 \times 10^{6} / \mathrm{ml}$ had a poorer prognosis compared with patients with Plt counts of $<400 \times 10^{6} / \mathrm{ml}$. Plt count and $\mathrm{Hb}$ level are easily measured, and are thus useful in periodical follow-up as prognostic factors for cervical cancer. The effectiveness of these biomarkers is shown in Table III.

Cytodiagnosis is used for cervical cancer and conventional tumor markers, including SCC, have little significance in early diagnosis. However, these markers are often useful for evaluating outcome, the extent of tumor spread and prediction of prognosis. SCC was developed by Kato and Torigoe (65) as a tumor-associated antigen in cervical cancer, and particularly in SCC, with positive rates of $2.44 \%$ in carcinoma in situ, $22.2 \%$ in FIGO stage I, 56.7\% in stage II, 76.4\% in stage III, and $76.4 \%$ in stage IV cervical SCC. Aberrantly high SCC levels in pretreatment serum in stages I and II may indicate a widespread tumor that is unresectable by radical hysterectomy (66), and this finding can affect the treatment strategy. Radical hysterectomy in patients with SCC-positive squamous cell cancer causes a rapid reduction in elevated SCC to undetectable levels within $72 \mathrm{~h}$ after surgery, whereas SCC levels remain elevated if an incomplete surgery, such as exploratory laparotomy or partial resection, is performed (67). For this reason, $\mathrm{SCC}$ is useful for postoperative evaluation.

Bolger et al (68) found that a serum SCC level of $\geq 8.6 \mathrm{mg} / \mathrm{ml}$ can predict lymph node metastasis with a positive predictive value of $100 \%$, and that micro-lymph node metastasis that cannot be detected by computed tomography can be predicted with low sensitivity. In 148 patients with stage IB cancer treated with surgery, Takeshima et al (69) found that $65 \%$ with preoperative serum SCC levels of $>4 \mathrm{ng} / \mathrm{ml}$ exhibited pelvic lymph node metastasis, and that this frequency was eight times higher than that in patients with preoperative serum SCC levels of $\leq 4 \mathrm{ng} / \mathrm{ml}$. SCC has a higher sensitivity than the marker cytokeratin 19 fragment 21-1 (CYFRA21-1), in squamous malignancies. However, CYFRA21-1 is more efficient than SCC for predicting lymph node metastasis and lymphovascular invasion (70). 
Table III. Biomarkers for cervical cancer.

\begin{tabular}{|c|c|}
\hline Biomarker & Effectiveness \\
\hline \multicolumn{2}{|c|}{ Associated with anticancer agent } \\
\hline CHFR & Prediction of sensitivity to paclitaxel \\
\hline WRN & Prediction of sensitivity to CPT-11 \\
\hline HIF- $1 \alpha$ & Prediction of sensitivity to topotecan \\
\hline EGFR & Prediction of sensitivity to anticancer agents \\
\hline D44v6, XRCC, mTOR & Prediction of sensitivity to platinum-type agents in neoadjuvant chemotherapy \\
\hline \multicolumn{2}{|c|}{ Associated with radiosensitivity } \\
\hline $\mathrm{COX}-2$ & Prediction of radiosensitivity \\
\hline HIF- $2 \alpha$ & Prediction of radiosensitivity \\
\hline \multicolumn{2}{|c|}{ Markers in peripheral blood } \\
\hline $\mathrm{Hb}, \mathrm{Plt}$ & Evaluation of prognosis \\
\hline $\mathrm{SCC}$ & Postoperative evaluation, prediction of lymph node metastasis \\
\hline CYFRA21-1 & Prediction of lymph node metastasis and vessel invasion \\
\hline CEA & Prediction of recurrence \\
\hline \multicolumn{2}{|l|}{ Others } \\
\hline TS & Evaluation of prognosis in advanced cervical cancer, prediction of radiosensitivity \\
\hline c-Myc & Staging of cervical cancer, evaluation of prognosis \\
\hline FHIT & Possible evaluation of prognosis \\
\hline GRP58 & Evaluation of prognosis in cervical adenocarcinoma \\
\hline MUC1, MUC16 & Evaluation of prognosis in cervical mucinous adenocarcinoma \\
\hline VEGF, PD-ECGF & Evaluation of prognosis, prediction of lymph node metastasis \\
\hline Dkk-3 & Evaluation of tumor diameter \\
\hline Ki-67 & Evaluation of prognosis \\
\hline CD109 & Targeted molecule in cervical SCC \\
\hline
\end{tabular}

CHFR, checkpoint with forkhead and ring finger; WRN, Werner DNA helicase; HIF-1 $\alpha$, hypoxia inducible factor $1 \alpha$; EGFR, epidermal growth factor receptor; XRCC, X-ray repair cross-complementing; COX-2, cyclooxygenase-2; Hb, hemoglobin; Plt, platelet; CYFRA21-1, cytokeratin 19 fragment 21-1; CEA, carcinoembryonic antigen; TS, thymidylate synthase; FHIT, fragile histidine triad; GRP58, glucose-related protein 58; VEGF, vascular endothelial growth factor; PD-ECGF, platelet-derived endothelial cell growth factor; Dkk-3, Dickkopf-3; CD109, cluster of differentiation 109; SCC, squamous cell carcinoma.

In patients with stage IB cervical cancer, Duk et al (71) found 5-year survival rates of $70 \%$ in cases with serum SCC of $\geq 2.5 \mathrm{ng} / \mathrm{ml}$, in contrast to $96 \%$ in patients with normal SCC levels. Thus, an SCC level of $\geq 2.5 \mathrm{ng} / \mathrm{ml}$ may be a prognostic factor. In 203 patients with stages IB1 to IV cervical cancer, Yamakawa et al (72) found significantly different 5-year survival rates of 39.4 and $79.0 \%$ in patients with SCC levels of $\geq 11.3$ and those $<11.3 \mathrm{ng} / \mathrm{ml}$, respectively. A plurality of lymph node metastases was found in patients with SCC levels of $\geq 11.3 \mathrm{ng} / \mathrm{ml}$, and the SCC level was indicated to be a factor for a poor prognosis. In 53 patients in stages III and IV, the 5 -year survival rates were 0 and $50 \%$ in cases with SCC levels of $\geq 25.5$ and those $<25.5 \mathrm{ng} / \mathrm{ml}$, respectively, and the significant difference between these groups indicates that aberrantly high SCC in stages III and IV can be used to determine the therapeutic strategy (72). Thus, SCC is useful for postoperative evaluation and prediction of lymph node metastasis, and is a significant prognostic factor in cervical cancer.

CYFRA21-1 is widely used as a tumor marker in the assessment of squamous malignancies. As aforementioned, CYFRA21-1 has a lower sensitivity than SCC in preoperative screening for cervical SCC, but may be more predictive for lymph node metastasis and lymphovascular invasion (70).

In pretreatment screening in patients with cervical SCC, carcinoembryonic antigen (CEA) is found at significantly different rates of 16.7 and $58.1 \%$ in cases without and with recurrence, respectively. Thus, CEA is an important tumor marker for predicting recurrence (Table III). te Velde et al (73) found a median time to recurrence in CEA-positive patients of $\sim 13$ weeks.

\section{Biomarkers for the prognostic prediction of cervical cancer}

c-Myc is a proto-oncogene that modulates cell proliferation through transcriptional regulation of genes required for proliferation. c-Myc is activated by genetic amplification during malignant transformation, with resultant overexpression at the mRNA and protein levels. Overexpression of c-Myc is involved in cervical cancer. Insertion of an HPV-DNA sequence close to the location of the c-Myc oncogene at 8q24.1 has been found in DNA extracted from cervical cancer tissue 
with c-Myc overexpression (74,75), suggesting that c-Myc transcription is activated by the integration of HPV-DNA in HPV-infected patients. Overexpression of c-Myc also occurs more frequently in patients with advanced cancer, and such patients have a significantly poorer survival compared with patients with normal c-Myc expression (76-78). Thus, evaluation of c-Myc expression is likely to be useful for the staging of cervical cancer and prognostic prediction.

FHIT is a cancer-suppressor gene that was identified by Ohta et al (79). Genetic alterations, including deletions and translocations, in the FHIT region occur in various malignancies and may be associated with carcinogenesis. Machida et al (80) investigated the association between FHIT expression and prognosis in 54 patients with stage IIIB cervical SCC who received radiation therapy as an initial treatment. There were no significant differences in age, radiation dose and para-aortic lymph node radiation between patients with abnormal and normal FHIT expression, while the abnormal FHIT expression was not associated with prognosis. In a study by Zhang et al (11), described in detail in the aforementioned CHFR section, FHIT methylation rates were not changed by silencing of DNMT1 in HeLa and SiHa cells. Thus, the association between FHIT and carcinogenesis is unclear and there is currently insufficient evidence to define FHIT as a prognostic factor in cervical cancer.

Liao et al (81) found that glucose-regulated protein 58 (GRP58) is a regulator of cell invasiveness and may function as a prognostic marker for cervical cancer. Overexpression of GRP58 was found in $73 \%$ of cervical cancer cases in screening for prognostic factors using 2D polyacrylamide gel electrophoresis, while immunohistochemical staining revealed that the histoscore for GRP58 was significantly elevated in patients with adenocarcinoma compared to those with SCC (81). Marked GRP58 staining was evident in adenocarcinoma with a penetration depth that was larger than half of the cervical stroma. A high GRP58 expression was significantly correlated with low survival rates and patients with overexpression of GRP58 and lymph node metastasis had poor outcomes. Thus, GRP58 is potentially a useful prognostic factor in cervical adenocarcinoma.

Togami et al (82) indicated that the expression of mucin antigens may be a prognostic factor in a study of 52 cases of cervical mucinous adenocarcinoma. The majority of cases had overexpression of MUC1 and MUC16 of mucin antigens, and these expression levels were associated with low survival rates. In particular, MUC1 overexpression was associated with a lower disease-free survival and greater lymph node metastasis, whereas the absence of expression of MUC1 and/or MUC16 was associated with longer overall and disease-free survival. Thus, these two mucin antigens are useful prognostic factors for cervical mucinous adenocarcinoma. Endometrial tumors have been shown to exhibit an increased expression of MUC1, MUC5B and MUC8 in comparison with normal tissues, however MUC1 is the only mucin antigen to be increased in cervical tumors (83).

Angiogenic factors are associated with the prognosis of cervical cancer as invasion and proliferation of tumor cells requires angiogenesis. In cervical cancer, the microvessel count in the tumor may also be an independent prognostic factor (84). Vascular endothelial growth factor (VEGF) is a typical angiogenic factor that is highly expressed in various types of cancer, and the level of VEGF is closely associated with the tumor microvessel count. VEGF-C, a VEGF family member, binds to VEGFR-3 in lymph vessels and promotes lymphangiogenesis (85). Lymph node metastasis is the main metastatic pathway, and thus, lymphangiogenesis is an important factor in this process. Cervical cancer patients with a high VEGF-C expression exhibit significant stromal invasion, lymph-vascular space involvement and lymph node metastasis. The multivariate analysis revealed that VEGF-C expression is an independent factor influencing lymph node metastasis (86). Platelet-derived endothelial cell growth factor (PD-ECGF) is an angiogenic factor derived from platelets. The expression level of PD-ECGF has also been associated with the tumor microvessel count in tumors in cervical cancer, uterine carcinoma and ovarian cancer (87). Fujimoto et al (88) showed that the prognosis of cases with a high PD-ECGF expression in metastatic lymph node lesions was extremely poor. Thus, angiogenic factors are closely associated with tumor invasion and proliferation, and are likely to have a significance as markers of prognosis.

Dickkopf-3 (Dkk-3) is a protein in the Dkk family. Jiang et al (89) found a significantly higher mean serum level of Dkk-3 in patients with cervical cancer $(166.39 \mathrm{pg} / \mathrm{ml})$ compared with healthy subjects $(42.08 \mathrm{pg} / \mathrm{ml})$. The serum levels of Dkk-3 in patients with cervical cancer were also associated with tumor diameters.

$\mathrm{Ki}-67$ is used clinically as a breast cancer proliferation marker. In patients with cervical SCC, Hanprasertpong et al (90) found $\mathrm{Ki}-67$ expression in $81.3 \%$ of cases and p53 expression in $33.6 \%$. There was a significant correlation between $\mathrm{p} 53$ and $\mathrm{Ki}-67$. The expression of Ki-67 was an independent prognostic factor for 5-year recurrence-free survival in multivariate analysis, whereas no prognostic significance of $\mathrm{p} 53$ expression was found. Shirendeb et al (91) found that expression levels of Ki-67 and p63 were significantly higher in HPV-16-positive patients compared with HPV-16-negative patients. Cimpean et al (92) showed that a lack of CD105/Ki-67 coexpression in endothelial cells was associated with the histopathology of the uterine cervix lesion and tumor proliferative status.

Zhang et al (93) found a significantly higher CD109 expression in cervical SCC compared with endometrial adenocarcinomas, normal cervix and endometrium. These findings indicate that CD109 may be a molecular target for the treatment of cervical SCC.

\section{Conclusion}

Novel treatment with greater efficacy than conventional therapies for advanced cervical cancer is likely to be established. Standard therapies can achieve a particular outcome in a cohort, but it is difficult to evaluate pretreatment sensitivity to anticancer agents and radiotherapy in each patient for selection of the optimal treatment approach. However, methods for treatment planning, evaluation following surgery and prediction of survival are important for determining a treatment strategy, particularly for advanced cervical cancer. Personalized treatment may be possible through use of biomarkers for sensitivity to anticancer agents, radiation, adverse reactions and treatment effects from an early stage. This approach is likely to 
produce an optimal treatment strategy and improve outcomes in patients with cervical cancer.

\section{Acknowledgements}

We thank Dr T. Tanaka and Dr R. Imai for their helpful assistance. The authors gratefully acknowledge grant support from the Japan Society for the Promotion of Science (JSPS) through a Grant-in-Aid for Scientific Research (KAKENHI), a Grant-in-Aid for Scientific Research (C) (22591866), and a Grant-in-Aid for Young Scientists (B) (24791718); the Medical Research Encouragement Prize of The Japan Medical Association; and the Keio Gijyuku Academic Development Fund. The funders had no role in data collection.

\section{References}

1. Greenlee RT, Murray T, Bolden S and Wingo PA: Cancer statistics, 2000. CA Cancer J Clin 50: 7-33, 2000.

2. Ushijima K: Current status of gynecologic cancer in Japan. J Gynecol Oncol 20: 67-71, 2009.

3. Soonthornthum T, Arias-Pulido H, Joste N, et al: Epidermal growth factor receptor as a biomarker for cervical cancer. Ann Oncol 22: 2166-2178, 2011.

4. Noordhuis MG, Eijsink JJ, Ten Hoor KA, et al: Expression of epidermal growth factor receptor (EGFR) and activated EGFR predict poor response to (chemo)radiation and survival in cervical cancer. Clin Cancer Res 15: 7389-7397, 2009.

5. Gadducci A, Guerrieri ME and Greco C: Tissue biomarkers as prognostic variables of cervical cancer. Crit Rev Oncol Hematol 86: 104-129, 2013

6. Noordhuis MG, Eijsink JJ, Roossink F, et al: Prognostic cell biological markers in cervical cancer patients primarily treated with (chemo)radiation: a systematic review. Int J Radiat Oncol Biol Phys 79: 325-334, 2011.

7. Sanbhnani S and Yeong FM: CHFR: a key checkpoint component implicated in a wide range of cancers. Cell Mol Life Sci 69: $1669-1687,2012$

8. Banno K, Yanokura M, Kawaguchi M, et al: Epigenetic inactivation of the CHFR gene in cervical cancer contributes to sensitivity to taxanes. Int J Oncol 31: 713-720, 2007.

9. Thigpen JT, Blessing JA, Fowler WC Jr and Hatch K: Phase II trials of cisplatin and piperazinedione as single agents in the treatment of advanced or recurrent non-squamous cell carcinoma of the cervix: a Gynecologic Oncology Group Study. Cancer Treat Rep 70: 1097-1100, 1986.

10. Papadimitriou CA, Sarris K, Moulopoulos LA, et al : Phase II trial of paclitaxel and cisplatin in metastatic and recurrent carcinoma of the uterine cervix. J Clin Oncol 17: 761-766, 1999.

11. Zhang Y, Chen FQ, Sun YH, Zhou SY, Li TY and Chen R: Effects of DNMT1 silencing on malignant phenotype and methylated gene expression in cervical cancer cells. J Exp Clin Cancer Res 30: 98, 2011.

12. Peng DF, Kanai Y, Sawada M, et al: DNA methylation of multiple tumor-related genes in association with overexpression of DNA methyltransferase 1 (DNMT1) during multistage carcinogenesis of the pancreas. Carcinogenesis 27: 1160-1168, 2006.

13. Liu N, Zhao LJ, Li XP, Wang JL, Chai GL and Wei LH: Histone deacetylase inhibitors inducing human cervical cancer cell apoptosis by decreasing DNA-methyltransferase 3B. Chin Med J (Engl) 125: 3273-3278, 2012.

14. Tadokoro T, Rybanska-Spaeder I, Kulikowicz T, et al: Functional deficit associated with a missense Werner syndrome mutation. DNA Repair (Amst) 12: 414-421, 2013.

15. Masuda K, Banno K, Yanokura M, et al: Association of epigenetic inactivation of the WRN gene with anticancer drug sensitivity in cervical cancer cells. Oncol Rep 28: 1146-1152, 2012.

16. Futami K, Takagi M, Shimamoto A, Sugimoto M and Furuichi Y: Increased chemotherapeutic activity of camptothecin in cancer cells by siRNA-induced silencing of WRN helicase. Biol Pharm Bull 30: 1958-1961, 2007.

17. Agrelo R, Cheng WH, Setien F, et al: Epigenetic inactivation of the premature aging Werner syndrome gene in human cancer. Proc Natl Acad Sci USA 103: 8822-8827, 2006.
18. Höpfl G,Ogunshola O and Gassmann M: HIFs and tumors - causes and consequences. Am J Physiol Regul Integr Comp Physiol 286: R608-R623, 2004.

19. Liao D and Johnson RS: Hypoxia: a key regulator of angiogenesis in cancer. Cancer Metastasis Rev 26: 281-290, 2007.

20. Vaupel P: Tumor microenvironmental physiology and its implications for radiation oncology. Semin Radiat Oncol 14: 198-206, 2004.

21. Michieli P: Hypoxia, angiogenesis and cancer therapy: to breathe or not to breathe? Cell Cycle 8: 3291-3296, 2009.

22. Sasabe E, Zhou X, Li D, Oku N, Yamamoto T and Osaki T: The involvement of hypoxia-inducible factor-1alpha in the susceptibility to gamma-rays and chemotherapeutic drugs of oral squamous cell carcinoma cells. Int J Cancer 120: 268-277, 2007.

23. Smith-McCune KK and Weidner N: Demonstration and characterization of the angiogenic properties of cervical dysplasia. Cancer Res 54: 800-804, 1994.

24. Mazibrada J, Rittà M, Mondini M, et al: Interaction between inflammation and angiogenesis during different stages of cervical carcinogenesis. Gynecol Oncol 108: 112-120, 2008.

25. Lee WY, Huang SC, Hsu KF, Tzeng CC and Shen WL: Roles for hypoxia-regulated genes during cervical carcinogenesis: somatic evolution during the hypoxia-glycolysis-acidosis sequence. Gynecol Oncol 108: 377-384, 2008.

26. Hutchison GJ, Valentine HR, Loncaster JA, et al: Hypoxia-inducible factor 1alpha expression as an intrinsic marker of hypoxia: correlation with tumor oxygen, pimonidazole measurements, and outcome in locally advanced carcinoma of the cervix. Clin Cancer Res 10: 8405-8412, 2004.

27. Burri P, Djonov V, Aebersold DM, et al: Significant correlation of hypoxia-inducible factor-1alpha with treatment outcome in cervical cancer treated with radical radiotherapy. Int J Radiat Oncol Biol Phys 56: 494-501, 2003.

28. Bachtiary B, Schindl M, Pötter R, et al: Overexpression of hypoxia-inducible factor 1alpha indicates diminished response to radiotherapy and unfavorable prognosis in patients receiving radical radiotherapy for cervical cancer. Clin Cancer Res 9: 2234-2240, 2003.

29. Bai H, Ge S, Lu J, Qian G and Xu R: Hypoxia inducible factor-1 $\alpha$-mediated activation of survivin in cervical cancer cells. J Obstet Gynaecol Res 39: 555-563, 2013.

30. Li R, Zhou L and Liu Q: Effect of different animal skin on the transdermal speed constant of sinomenine. Zhong Yao Cai 21: 580-583, 1998 (In Chinese).

31. Luczak MW, Roszak A, Pawlik P, et al: Transcriptional analysis of CXCR4, DNMT3A, DNMT3B and DNMT1 gene expression in primary advanced uterine cervical carcinoma. Int J Oncol 40: 860-866, 2012

32. Kummar S, Raffeld M, Juwara L, et al: Multihistology, target-driven pilot trial of oral topotecan as an inhibitor of hypoxia-inducible factor- $1 \alpha$ in advanced solid tumors. Clin Cancer Res 17: 5123-5131, 2011.

33. Rapisarda A, Shoemaker RH and Melillo G: Targeting topoisomerase I to inhibit hypoxia inducible factor 1. Cell Cycle 3: 172-175, 2004.

34. Rapisarda A, Uranchimeg B, Scudiero DA, et al: Identification of small molecule inhibitors of hypoxia-inducible factor 1 transcriptional activation pathway. Cancer Res 62: 4316-4324, 2002.

35. Rapisarda A, Uranchimeg B, Sordet O, Pommier Y, Shoemaker RH and Melillo G: Topoisomerase I-mediated inhibition of hypoxia-inducible factor 1: mechanism and therapeutic implications. Cancer Res 64: 1475-1482, 2004.

36. Hynes NE and Lane HA: ERBB receptors and cancer: the complexity of targeted inhibitors. Nat Rev Cancer 5: 341-354, 2005.

37. Yarden Y and Sliwkowski MX: Untangling the ErbB signalling network. Nat Rev Mol Cell Biol 2: 127-137, 2001.

38. Liang K, Ang KK, Milas L, Hunter N and Fan Z: The epidermal growth factor receptor mediates radioresistance. Int J Radiat Oncol Biol Phys 57: 246-254, 2003.

39. Milas L, Fan Z, Andratschke NH and Ang KK: Epidermal growth factor receptor and tumor response to radiation: in vivo preclinical studies. Int J Radiat Oncol Biol Phys 58: 966-971, 2004.

40. Halle C, Lando M, Svendsrud DH, et al: Membranous expression of ectodomain isoforms of the epidermal growth factor receptor predicts outcome after chemoradiotherapy of lymph node-negative cervical cancer. Clin Cancer Res 17: 5501-5512, 2011.

41. Iida K, Nakayama K, Rahman MT, et al: EGFR gene amplification is related to adverse clinical outcomes in cervical squamous cell carcinoma, making the EGFR pathway a novel therapeutic target. Br J Cancer 105: 420-427, 2011 
42. Schrevel M, Karim R, ter Haar NT, et al: CXCR7 expression is associated with disease-free and disease-specific survival in cervical cancer patients. Br J Cancer 106: 1520-1525, 2012.

43. Monk BJ, Mas Lopez L, Zarba JJ, et al: Phase II, open-label study of pazopanib or lapatinib monotherapy compared with pazopanib plus lapatinib combination therapy in patients with advanced and recurrent cervical cancer. J Clin Oncol 28: 3562-3569, 2010.

44. Costa S, Terzano P, Bovicelli A, et al: CD44 isoform 6 (CD44v6) is a prognostic indicator of the response to neoadjuvant chemotherapy in cervical carcinoma. Gynecol Oncol 80: 67-73, 2001.

45. Chung HH, Kim MK, Kim JW, et al: XRCC1 R399Q polymorphism is associated with response to platinum-based neoadjuvant chemotherapy in bulky cervical cancer. Gynecol Oncol 103: 1031-1037, 2006.

46. Faried LS, Faried A, Kanuma T, et al: Predictive and prognostic role of activated mammalian target of rapamycin in cervical cancer treated with cisplatin-based neoadjuvant chemotherapy. Oncol Rep 16: 57-63, 2006.

47. Gupta RA and Dubois RN: Colorectal cancer prevention and treatment by inhibition of cyclooxygenase-2. Nat Rev Cancer 1: 11-21, 2001.

48. Zhang F, Engebretson SP, Morton RS, Cavanaugh PF Jr, Subbaramaiah K and Dannenberg AJ: The overexpression of cyclo-oxygenase-2 in chronic periodontitis. J Am Dent Assoc 134: 861-867, 2003.

49. Katori M and Majima M: Cyclooxygenase-2: its rich diversity of roles and possible application of its selective inhibitors. Inflamm Res 49: 367-392, 2000.

50. Kulkarni S, Rader JS, Zhang F, et al: Cyclooxygenase-2 is overexpressed in human cervical cancer. Clin Cancer Res 7: 429-434, 2001.

51. Raju U, Ariga H, Dittmann K, Nakata E, Ang KK and Milas L: Inhibition of DNA repair as a mechanism of enhanced radioresponse of head and neck carcinoma cells by a selective cyclooxygenase-2 inhibitor, celecoxib. Int J Radiat Oncol Biol Phys 63: 520-528, 2005.

52. Xia S, Zhao Y, Yu S and Zhang M: Activated PI3K/Akt/COX-2 pathway induces resistance to radiation in human cervical cancer HeLa cells. Cancer Biother Radiopharm 25: 317-323, 2010.

53. Wang AH, Tian XY, Yu JJ, Mi JQ, Liu H and Wang RF: Celecoxib radiosensitizes the human cervical cancer HeLa cell line via a mechanism dependent on reduced cyclo-oxygenase-2 and vascular endothelial growth factor $\mathrm{C}$ expression. J Int Med Res 40: 56-66, 2012.

54. Ferrandina G, Ranelletti FO, Legge F, et al: Prognostic role of the ratio between cyclooxygenase- 2 in tumor and stroma compartments in cervical cancer. Clin Cancer Res 10: 3117-3123, 2004.

55. Kim YB, Kim GE, Pyo HR, et al: Differential cyclooxygenase-2 expression in squamous cell carcinoma and adenocarcinoma of the uterine cervix. Int J Radiat Oncol Biol Phys 60: 822-829, 2004.

56. Kim GE, Kim YB, Cho NH, et al: Synchronous coexpression of epidermal growth factor receptor and cyclooxygenase- 2 in carcinomas of the uterine cervix: a potential predictor of poor survival. Clin Cancer Res 10: 1366-1374, 2004.

57. Suzuki M, Tsukagoshi S, Saga Y, Ohwada M and Sato I: Enhanced expression of thymidylate synthase may be of prognostic importance in advanced cervical cancer. Oncology 57: 50-54, 1999.

58. Kawanaka T, Kubo A, Ikushima H, Sano T, Takegawa $Y$ and Nishitani H: Prognostic significance of HIF-2alpha expression on tumor infiltrating macrophages in patients with uterine cervical cancer undergoing radiotherapy. J Med Invest 55: 78-86, 2008.

59. Blancher C, Moore JW, Talks KL, Houlbrook S and Harris AL: Relationship of hypoxia-inducible factor (HIF)-1alpha and HIF-2alpha expression to vascular endothelial growth factor induction and hypoxia survival in human breast cancer cell lines. Cancer Res 60: 7106-7113, 2000.

60. Ziemer LS, Koch CJ, Maity A, Magarelli DP, Horan AM and Evans SM: Hypoxia and VEGF mRNA expression in human tumors. Neoplasia 3: 500-508, 2001

61. Vaupel P, Kelleher DK and Höckel M: Oxygen status of malignant tumors: pathogenesis of hypoxia and significance for tumor therapy. Semin Oncol 28 (Suppl 8): 29-35, 2001.

62. Girinski T, Pejovic-Lenfant MH, Bourhis J, et al: Prognostic value of hemoglobin concentrations and blood transfusions in advanced carcinoma of the cervix treated by radiation therapy: results of a retrospective study of 386 patients. Int J Radiat Oncol Biol Phys 16: 37-42, 1989.
63. Thomas G: The effect of hemoglobin level on radiotherapy outcomes: the Canadian experience. Semin Oncol 28 (Suppl 8): 60-65, 2001.

64. Hernandez E, Donohue KA, Anderson LL, Heller PB and Stehman FB: The significance of thrombocytosis in patients with locally advanced cervical carcinoma: a Gynecologic Oncology Group study. Gynecol Oncol 78: 137-142, 2000.

65. Kato $\mathrm{H}$ and Torigoe T: Radioimmunoassay for tumor antigen of human cervical squamous cell carcinoma. Cancer 40: 1621-1628, 1977.

66. Kato H, Morioka H, Tsutsui H, Aramaki S and Torigoe T: Value of tumor-antigen (TA-4) of squamous cell carcinoma in predicting the extent of cervical cancer. Cancer 50: 1294-1296, 1982.

67. Maruo T, Shibata K, Kimura A, Hoshina M and Mochizuki M: Tumor-associated antigen, TA-4, in the monitoring of the effects of therapy for squamous cell carcinoma of the uterine cervix. Serial determinations and tissue localization. Cancer 56: 302-308, 1985.

68. Bolger BS, Dabbas M, Lopes A and Monaghan JM: Prognostic value of preoperative squamous cell carcinoma antigen level in patients surgically treated for cervical carcinoma. Gynecol Oncol 65: 309-313, 1997.

69. Takeshima N, Hirai Y, Katase K, Yano K, Yamauchi K and Hasumi K: The value of squamous cell carcinoma antigen as a predictor of nodal metastasis in cervical cancer. Gynecol Oncol 68: 263-266, 1998.

70. Puthucode-Easwaran S, Naik R, Athavale R, et al: Comparison of pre-treatment CYFRA 21-1 and SCC-Antigen assay in primary cervical carcinoma - a preliminary report. J Obstet Gynaecol 25: 486-488, 2005.

71. Duk JM, de Bruijn HW, Groenier KH, et al: Cancer of the uterine cervix: sensitivity and specificity of serum squamous cell carcinoma antigen determinations. Gynecol Oncol 39: 186-194, 1990.

72. Yamakawa H, Konno R, Enomoto A, et al: Prognostic factors of uterine cervical cancer. Gan To Kagaku Ryoho 33: 2002-2007, 2006 (In Japanese).

73. te Velde ER, Persijn JP, Ballieux RE and Faber J: Carcinoembryonic antigen serum levels in patients with squamous cell carcinoma of the uterine cervix: clinical significance. Cancer 49: 1866-1873, 1982.

74. Hori T, Ichimura H, Minamihisamatsu M, et al: Chromosomal insertion and amplification of human papillomavirus 16 DNA sequences in a cell line of argyrophil small cell carcinoma of the uterine cervix. Jpn J Cancer Res 82: 371-375, 1991.

75. Couturier J, Sastre-Garau X, Schneider-Maunoury S, Labib A and Orth G: Integration of papillomavirus DNA near myc genes in genital carcinomas and its consequences for proto-oncogene expression. J Virol 65: 4534-4538, 1991.

76. Iwasaka T, Yokoyama M, Oh-uchida M, et al: Detection of human papillomavirus genome and analysis of expression of c-myc and Ha-ras oncogenes in invasive cervical carcinomas. Gynecol Oncol 46: 298-303, 1992.

77. Pinion SB, Kennedy JH, Miller RW and MacLean AB: Oncogene expression in cervical intraepithelial neoplasia and invasive cancer of cervix. Lancet 337: 819-820, 1991.

78. Riou G, Lê MG, Favre M, Jeannel D, Bourhis J and Orth G: Human papillomavirus-negative status and c-myc gene overexpression: independent prognostic indicators of distant metastasis for early-stage invasive cervical cancers. J Natl Cancer Inst 84: 1525-1526, 1992.

79. Ohta M, Inoue H, Cotticelli MG, et al: The FHIT gene, spanning the chromosome 3 p14.2 fragile site and renal carcinoma-associated $\mathrm{t}(3 ; 8)$ breakpoint, is abnormal in digestive tract cancers. Cell 84: 587-597, 1996.

80. Machida S, Ohwada M, Saga Y and Suzuki M: Abnormal fragile histidine triad expression in advanced cervical cancer and evaluation of its utility as a prognostic factor. Oncology 65 : 89-93, 2003.

81. Liao CJ, Wu TI, Huang YH, et al: Glucose-regulated protein 58 modulates cell invasiveness and serves as a prognostic marker for cervical cancer. Cancer Sci 102: 2255-2263, 2011.

82. Togami S, Nomoto M, Higashi M, et al: Expression of mucin antigens (MUC1 and MUC16) as a prognostic factor for mucinous adenocarcinoma of the uterine cervix. J Obstet Gynaecol Res 36: 588-597, 2010.

83. Hebbar V, Damera G and Sachdev GP: Differential expression of MUC genes in endometrial and cervical tissues and tumors. BMC Cancer 5: 124, 2005. 
84. Kodama J, Yoshinouchi M, Seki N, Hongo A, Miyagi Y and Kudo T: Angiogenesis and platelet-derived endothelial cell growth factor/thymidine phosphorylase expression in cervical cancer. Int J Oncol 15: 149-154, 1999.

85. Jussila L and Alitalo K: Vascular growth factors and lymphangiogenesis. Physiol Rev 82: 673-700, 2002.

86. Hashimoto I, Kodama J, Seki N, et al: Vascular endothelial growth factor-C expression and its relationship to pelvic lymph node status in invasive cervical cancer. Br J Cancer 85: 93-97, 2001.

87. Ishikawa F, Miyazono K, Hellman U, et al: Identification of angiogenic activity and the cloning and expression of platelet-derived endothelial cell growth factor. Nature 338: 557-562, 1989.

88. Fujimoto J, Sakaguchi H, Hirose R, Wen H and Tamaya T: Clinical implication of expression of platelet-derived endothelial cell growth factor (PD-ECGF) in metastatic lesions of uterine cervical cancers. Cancer Res 59: 3041-3044, 1999.

89. Jiang T, Huang L, Wang S and Zhang S: Clinical significance of serum Dkk-3 in patients with gynecological cancer. J Obstet Gynaecol Res 36: 769-773, 2010.
90. Hanprasertpong J, Tungsinmunkong K, Chichareon S, et al: Correlation of p53 and Ki-67 (MIB-1) expressions with clinicopathological features and prognosis of early stage cervical squamous cell carcinomas. J Obstet Gynaecol Res 36: 572-580, 2010.

91. Shirendeb U, Hishikawa Y, Moriyama S, et al: Human papillomavirus infection and its possible correlation with p63 expression in cervical cancer in Japan, Mongolia, and Myanmar. Acta Histochem Cytochem 42: 181-190, 2009.

92. Cimpean AM, Saptefrati L, Ceausu R and Raica M: Characterization of endoglin and Ki-67 expression in endothelial cells from benign and malignant lesions of the uterine cervix. Pathol Int 59: 695-700, 2009.

93. Zhang JM, Hashimoto M, Kawai K, et al: CD109 expression in squamous cell carcinoma of the uterine cervix. Pathol Int 55: 165-169, 2005. 